\title{
UNBOUNDED GROWTH OF ENERGY IN PERIODIC PERTURBATIONS OF GEODESIC FLOWS OF THE TORUS
}

\author{
AMADEU DELSHAMS \\ Departament de Matemàtica Aplicada I, Universitat Politècnica de Catalunya, \\ Diagonal 647, 08028 Barcelona, Spain, amadeu@ma1.upc.es \\ RAFAEL DE LA LLAVE \\ Department of Mathematics, University of Texas at Austin, Austin, TX, 78712, \\ $U S A$, llave@math.utexas.edu \\ TERE M. SEARA \\ Departament de Matemàtica Aplicada I, Universitat Politècnica de Catalunya, \\ Diagonal 647, 08028 Barcelona, Spain, tere@ma1.upc.es
}

\section{Introduction}

The goal of these notes is to summarize the main ideas of a paper by the authors. ${ }^{1}$ We will study the effects of a periodic external potential on the dynamics of the geodesic flow in the (2-dimensional) torus. This is a Hamiltonian system with two and a half degrees of freedom with Hamiltonian $H(p, q, t)=\frac{1}{2} g_{q}(p, p)+U(q, t)$. Here $g$ is the metric on the torus, and $U$ is the external periodic potential.

If $U=0$, the system is autonomous and, therefore, the energy of the geodesic flow $H_{0}(p, q)=\frac{1}{2} g_{q}(p, p)$ is preserved. Note also that when the metric is flat, the geodesic flow is integrable.

We will establish, using geometric methods, a result that had been established by J. Mather using variational methods. ${ }^{2}$ Namely, that for generic metrics and potentials - in particular for arbitrarily small potentials and for metrics arbitrarily close to integrable-, one can find orbits whose energy grows to infinity.

More precisely, we will indicate the proof of:

Theorem 1 For every $r=15, \ldots, \infty, \omega$, there exists a $\mathcal{C}^{r}$-residual set in the set of metrics $g$ on $\mathbb{T}^{2}$, and of periodic potentials $U: \mathbb{T}^{2} \times \mathbb{T}^{1} \rightarrow \mathbb{R}$, such that the Hamiltonian $H(p, q, t)=\frac{1}{2} g_{q}(p, p)+U(q, t)$ has an orbit of unbounded energy.

The result is somewhat more surprising if we realize that, for high energy, the potential is an small perturbation of the geodesic flow and that since there are two time scales involved (see subsection 2.2), the frequency of the 
perturbation is smaller than the frequency of the geodesic flow.

In this sense this result can be understood as an analogue to Arnold diffusion. The difference between this work and other approaches to Arnold diffusion in the literature comes from the fact that here, the unperturbed system, which is the geodesic flow, is a two degrees of freedom Hamiltonian system that, in general, is not integrable and it is assumed to have some hyperbolicity properties. Indeed, the hyperbolicity properties involve that the system contains hyperbolic sets with transversal intersection in an energy surface. This is somewhat stronger hyperbolicity that the so-called a-priori unstable systems in the notation of Chierchia and Gallavotti ${ }^{3}$.

We propose the name a-priori chaotic for this kind of systems, where there are some conserved quantities, but there are hyperbolic orbits with transverse heteroclinic intersections in the manifolds corresponding to the conserved quantities.

This result on instability has been proved by J. Mather using a variational approach (the differentiability requirements were much smaller). The proof presented here will rely on standard tools in the study of dynamical systems. Most of them have been used traditionally in Arnold diffusion. We also use very strongly the theory of hyperbolic dynamical systems. We hope that this can serve as a clarification of the relation between variational methods and more geometric methods. We also point out that S. Bolotin and D. Treschev have presented another geometric proof ${ }^{4}$. The main difference with the method presented here is that they rely more on KAM theory and they do not use the theory of normally hyperbolic manifolds.

The generic conditions that we need can be described rather explicitly. We require for the metric that the geodesic flow possesses a hyperbolic periodic orbit with a homoclinic orbit. Once we fix this periodic orbit and the homoclinic connection, we require on the potential that the Poincare function $\mathcal{L}(\tau)$ computed on it is not identically constant (see (24)).

The proof can be divided in several steps that are largely independent. As we mentioned before, each step only requires almost standard tools. Let us go over a thumbnail sketch of all these steps and they will be fleshed out in the following sections.

The same method of proof allows to prove somewhat more general results. If we assume the consequences of one step, then all the subsequent ones remain valid. Moreover, we can use the same method to obtain more consequences. Indeed, the proof will be established by the somewhat more general Theorem 18. 


\section{Riemannian Geometry}

It was shown already by Morse - with some improvements by Mather-that a generic metric possesses a hyperbolic closed geodesic with a homoclinic orbit.

Note that this shows that the geodesic flow is not integrable and is a-priori unstable in the sense of Chierchia and Gallavotti. ${ }^{3}$

It is a crucial observation that, when we consider the geodesic flow as a 4-dimensional dynamical system, a closed geodesic lifts to a cylinder $\Lambda$ (one periodic orbit for each energy surface). This cylinder is invariant and normally hyperbolic.

\section{Scaled variables}

As can be seen by elementary dimensional analysis, if we rescale the momenta and the time, we can transform the perturbation introduced by $U$ for high energy as an small and slow perturbation of the geodesic flow. Intuitively, if the geodesics are moving very fast, in their time scale, the potential is changing slowly.

For the analysis that will follow, the slowness is much more important than the smallness.

Another elementary remark is that we can consider the system as autonomous by considering one extra angle variable. We can come back from this extended phase space to the regular phase space by means of the Poincaré return map. In particular, the cylinder $\Lambda$ is invariant under the action of the unperturbed Poincaré map, and it lifts to a 3-dimensional manifold $\tilde{\Lambda}$, invariant under the unperturbed extended flow.

In this sketch, we will be somewhat cavalier about distinguishing between the objects in the extended phase space and in the regular phase space.

The theory of normally hyperbolic manifolds

Since $\tilde{\Lambda}$ is uniformly hyperbolic in the extended phase space, we can find a manifold close to it that is invariant for the perturbed system.

The stable and unstable manifolds of this invariant manifold still intersect as transversally as possible.

The importance of this invariant manifold is that it will serve as a template for many types of orbits. 


\section{Averaging theory}

Taking advantage of the fact that the perturbation is slow, we can study the motion on the perturbed invariant manifold using the method of averaging.

Assuming that the system is differentiable enough, we can perform several steps of averaging so that the system on the 3 -dimensional invariant manifold is a very small periodic perturbation of an integrable Hamiltonian system.

\section{KAM theory}

Once we know that a system is close to an integrable Hamiltonian system, we can find out that it is covered very densely by invariant tori, emerging from invariant circles of the Poincaré map.

\section{Poincaré-Melnikov theory}

It turns out to be possible to compute perturbatively the gain in energy for a homoclinic excursion.

Under some generic hypotheses, it is possible to show that a homoclinic excursion leads to a jump from one KAM torus to another, giving rise to heteroclinic orbits between them.

This idea can be formulated precisely by introducing what we call the scattering map which relates the asymptotics in the future with the asymptotics in the past. This map can be constructed precisely using hyperbolicity theory and it can be computed perturbatively using the Poincaré-Melnikov method (the fundamental theorem of calculus and a - somewhat delicate- passage to the limit).

Under the assumption that the scattering map moves enough to jump over the gaps between KAM tori-which we have already shown are very small-we can construct pseudo-orbits whose energy grows unboundedly.

These pseudorbits can be described as follows. When the potential is in such a condition that produces a jump of energy in the direction that we want, we execute the jump, following a heteroclinic orbit, and in the situations when it is not favorable, we just bid our time near a KAM torus.

\section{Shadowing lemma}

We need to construct orbits that follow these pseudo-orbits closely.

This can be done by a topological argument very close to the original one invoking the obstruction property. ${ }^{5,6}$

Now, we turn to the task of fleshing out this sketch. 


\section{The main steps of the proof}

\subsection{Riemannian geometry and dynamics of the geodesic flow}

Let us begin by describing the dynamics of the geodesic flow. The phase space of the geodesic flow is $\mathbf{T}^{*} \mathbb{T}^{2}=\mathbb{R}^{2} \times \mathbb{T}^{2}$. We will denote the coordinates in $\mathbb{T}^{2}$ by $q$ and the cotangent directions by $p$. The geodesic flow is a two degrees of freedom Hamiltonian, with Hamiltonian $H_{0}(p, q)=\frac{1}{2} g_{q}(p, p)$, with respect to the canonical symplectic form $\Omega$, which, moreover, is exact: $\Omega=d \theta$. In local coordinates, $\theta=\sum_{i} p_{i} d q_{i}, \Omega=\sum_{i} d p_{i} \wedge d q_{i}$. We will denote by $\Phi(t ; p, q)=\Phi_{t}(p, q)$ this geodesic flow, and we remark that, even though this flow is not integrable, the energy $E=H_{0}$ is preserved.

For each $E$, we will denote $\Sigma_{E}=\left\{(p, q): H_{0}(p, q)=E\right\}$, which is a 3dimensional manifold diffeomorphic to $\mathbb{T}^{1} \times \mathbb{T}^{2}$, invariant under the geodesic flow. We observe that, for any $E_{0}>0, \tilde{\Sigma}_{E_{0}}=\cup_{E \geq E_{0}} \Sigma_{E} \simeq\left[E_{0}, \infty\right) \times \mathbb{T}^{1} \times \mathbb{T}^{2}$, that is, the phase space is foliated by the energy surfaces.

Given an arbitrary geodesic " $\lambda$ " : $\mathbb{R} \rightarrow \mathbb{T}^{2}$, parameterized by arc length, we will denote by $\lambda_{E}(t)=\left(\lambda_{E}^{p}(t), \lambda_{E}^{q}(t)\right)$ the orbit of the geodesic flow that lies in the energy surface $\Sigma_{E}$, and whose projection over $q$ turns along the range of " $\lambda$ ". Moreover, we fix the origin of time in $\lambda_{E}$ so that it corresponds to the origin of the parameterization in " $\lambda$ ". (Formally $H_{0}\left(\lambda_{E}(t)\right)=E$, and Range $(" \lambda ")=\operatorname{Range}\left(\lambda_{E}^{q}\right)$, " $\lambda$ " $(0)=\lambda_{E}^{q}(0)$.)

Note that

$$
\left(\lambda_{E}^{p}(t), \lambda_{E}^{q}(t)\right)=\left(\sqrt{2 E} \lambda_{1 / 2}^{p}(\sqrt{2 E} t), \lambda_{1 / 2}^{q}(\sqrt{2 E} t)\right) .
$$

So that, in this situation, the role of $E$ is just a rescaling of time. The following theorem is due to Morse and Mather:

Theorem 2 For a $\mathcal{C}^{r}$ generic metric $g, r \geq 2$, and for any value of the Hamiltonian $H_{0}(p, q)=E>0$, there exists a periodic orbit $\Lambda_{E}(t)$, as in (1), of the Hamiltonian flow associated to $H_{0}$ whose range $\hat{\Lambda}_{E}$ is a normally hyperbolic invariant manifold in the energy surface. Its stable and unstable manifolds $W_{\hat{\Lambda}_{E}}^{\text {,u }}$ are 2-dimensional, and there exists a homoclinic orbit $\gamma_{E}(t)$ such that its range $\hat{\gamma}_{E}$ satisfies

$$
\hat{\gamma}_{E} \subset\left(W_{\hat{\Lambda}_{E}}^{\mathrm{s}} \backslash \hat{\Lambda}_{E}\right) \cap\left(W_{\hat{\Lambda}_{E}}^{\mathrm{u}} \backslash \hat{\Lambda}_{E}\right) .
$$

Moreover, this intersection is transverse as intersection of invariant manifolds in the energy surface along $\hat{\gamma}_{E}$.

For $E=1 / 2$, we have that

$$
\operatorname{dist}\left(\Lambda_{1 / 2}\left(t+a_{ \pm}\right), \gamma_{1 / 2}(t)\right) \rightarrow 0 \quad \text { as } \quad t \rightarrow \pm \infty \text {. }
$$


We suppose that the length of the unit geodesic " $\Lambda$ " on the metric $g$ is 1. Note that the speed of a unit geodesic is 1 and, therefore, its energy is $1 / 2$. Then " $\Lambda$ " as an orbit of the geodesic flow has period 1 and energy $1 / 2$. Because of this we use the notation $\Lambda_{1 / 2}$.

The quantity $\Delta \equiv a_{+}-a_{-}$is called phase shift. In this situation the energy is preserved and this makes the periodic orbit non-hyperbolic in the whole space. In order to obtain some hyperbolic object it is natural to discuss what happens for all energy surfaces considering $\Lambda=\bigcup_{E \geq E_{0}} \hat{\Lambda}_{E}$ for all values of the energy.

Lemma 3 Define $\Lambda=\bigcup_{E>E_{0}} \hat{\Lambda}_{E}$. This is a 2-dimensional manifold with boundary which is diffeomorphic to $\left[E_{0}, \infty\right) \times \mathbb{T}^{1}$, and the canonical symplectic form on $\mathbf{T}^{*} \mathbb{T}^{2} \Omega$ restricted to $\Lambda$ is non-degenerate and invariant under the geodesic flow $\Phi_{t}$.

The stable and unstable manifolds to $\Lambda, W_{\Lambda}^{\mathrm{s}}, W_{\Lambda}^{\mathrm{u}}$, are 3 -dimensional manifolds diffeomorphic to $\left[E_{0}, \infty\right) \times \mathbb{T}^{1} \times \mathbb{R}$, and $\gamma=\bigcup_{E \geq E_{0}} \hat{\gamma}_{E}$ is a 2-dimensional manifold diffeomorphic to $\left[E_{0}, \infty\right) \times \mathbb{R}$ that verifies

$$
\gamma \subset\left(W_{\Lambda}^{\mathrm{s}} \backslash \Lambda\right) \cap\left(W_{\Lambda}^{\mathrm{u}} \backslash \Lambda\right) .
$$

Let us note that the intersection of the stable and unstable manifolds along $\gamma$ is transverse, because

$$
T_{x} W_{\Lambda}^{\mathrm{s}} \cap T_{x} W_{\Lambda}^{\mathrm{u}}=T_{x} \gamma
$$

This manning of transversal intersection has several important consequences. For example, $\gamma$ will be a locally unique intersection.

Since the manifold $\Lambda$ is a cylinder, we are going to take a coordinate system in $\Lambda$, given by one real coordinate (momentum) and one angle (position). The real coordinate will be $J=\sqrt{2 H_{0}} \geq \sqrt{2 E_{0}}$. For the angle coordinate, we will take $\varphi \in \mathbb{T}^{1}$, which is determined by $d J \wedge d \varphi=\Omega \mid \Lambda$ and $\varphi=0$ corresponds to the origin of the parameterization in " $\Lambda$ ". We can identify a point in $\Lambda$ with the values $(J, \varphi)$ taken by these coordinates. The coordinate representation of the periodic orbit $\Lambda_{E}(t)$ in these coordinates is given by $(J, J t)$, where $J=\sqrt{2 E}$.

\subsection{The scaled problem}

In order to make the perturbative structure of the problem more apparent we will scale the variables $p$ and the time $t$ in the Hamiltonian $H(p, q, t)=$ $\frac{1}{2} g_{q}(p, p)+U(q, t)$.

We pick a (large) number $E_{*}$, and call $\varepsilon=1 / \sqrt{E_{*}}$. Since $\varepsilon^{2} H(p, q)=$ $\frac{1}{2} g_{q}(\varepsilon p, \varepsilon p)+\varepsilon^{2} U(q, t)$, we introduce $\bar{p}=\varepsilon p$ and consider the symplectic form 
$\bar{\Omega}=d \bar{p} \wedge d q=\varepsilon \Omega$, noting that $q, \bar{p}$ are conjugate variables in $\bar{\Omega}$. Next, we introduce a new time $\bar{t}=t / \varepsilon$, and we obtain a Hamiltonian system with Hamiltonian

$$
\bar{H}_{\varepsilon}(\bar{p}, q, \varepsilon \bar{t})=\frac{1}{2} g_{q}(\bar{p}, \bar{p})+\varepsilon^{2} U(q, \varepsilon \bar{t})=H_{0}(\bar{p}, q)+\varepsilon^{2} U(q, \varepsilon \bar{t}) .
$$

We also introduce $\bar{E}=E / E_{*}$, and we are going to see that, for sufficiently large $E_{*}$, there exist hyperbolic whiskered tori with heteroclinic orbits between them whose energies are between $\bar{E}=1 / 2+\delta$ and $\bar{E}=2-\delta$ for some small $\delta>0$. Since the result is for all sufficiently large $E_{*}$, we will be able to take a larger $E_{*}$ and continue the transition chain.

From now on and until further notice, we will drop the bar from the problem. We will refer to the bar variables as the rescaled variables and the original ones as the physical variables. Then the Hamiltonian $H_{\varepsilon}$ and all the functions derived from it will be $1 / \varepsilon$ periodic in time. In order to do this more apparent we will use the notation given in (3).

\subsection{The inner and outer maps}

We will define two maps in $\Lambda$ which describe, respectively, the inner dynamics in $\Lambda$ and the outer dynamics given by the homoclinic connections that lie in $\gamma$. In the unperturbed case the dynamics is known, and we will be able to give explicit expressions for these maps. Later on, when we consider the problem with external potential, the perturbative computation of these maps will be the main tool to control the behavior of the orbits.

The inner map $F_{0}$ will be the time $1 / \varepsilon$ map of the geodesic flow, also called Poincaré map, restricted to $\Lambda$. As we are dealing with the autonomous case, the phase portrait of this map is identical to that of the geodesic flow. For simplicity we will consider the Poincaré map associated to the initial time 0 , that is $F_{0}(x)=\Phi_{1 / \varepsilon}(x)$, where $\Phi_{t}(x)$ denotes the geodesic flow on the torus. We note that the behavior of this unperturbed map is exactly the same as the unperturbed flow, and then all the possible choices of the initial time give rise to the same map. Even though this map is defined in all the phase space, we are interested only on the dynamics in $\Lambda$, which, in the coordinates $(J, \varphi)$, is given by:

$$
F_{0}(J, \varphi)=(J, \varphi+J / \varepsilon) .
$$

We call attention to the fact that $F_{0}$ is a twist map and this will be useful to study the effects of the perturbation on it. We also note that the periodic orbits of the geodesic flow are invariant curves for this map, and they are given in these coordinates by $J=$ const. 
The outer map or "scattering map" $S_{0}: \Lambda \rightarrow \Lambda$, associated to $\gamma$, will transform the asymptotic point at $-\infty$ of an orbit homoclinic to $\Lambda$ into the asymptotic point at $+\infty$. Thus we define $x_{+}=S_{0}\left(x_{-}\right)$if there exists $z \in \gamma \subset$ $\mathbf{T}^{*} \mathbb{T}^{2}$, such that

$$
\operatorname{dist}\left(\Phi_{t}\left(x_{ \pm}\right), \Phi_{t}(z)\right) \rightarrow 0, \quad \text { as } \quad t \rightarrow \pm \infty .
$$

From the results of Morse and Mather of Theorem 2:

$$
\operatorname{dist}\left(\Lambda_{1 / 2}\left(t+a_{ \pm}\right), \gamma_{1 / 2}(t)\right) \rightarrow 0, \quad \text { as } t \rightarrow \pm \infty
$$

and the rescaling properties give

$$
\operatorname{dist}\left(\Lambda_{E}\left(t / \sqrt{2 E}+a_{ \pm} / \sqrt{2 E}\right), \gamma_{E}(t / \sqrt{2 E})\right) \rightarrow 0 \text { as } t \rightarrow \pm \infty .
$$

Hence, expressing the map $S_{0}$ in the coordinates $(J, \varphi)$,

$$
S_{0}\left(J, a_{-}+\varphi\right)=\left(J, a_{+}+\varphi\right),
$$

or more simply, remembering that $\Delta=a_{+}-a_{-}$is the phase shift:

$$
S_{0}(J, \varphi)=(J, \varphi+\Delta) .
$$

Now, in the unperturbed case, the dynamics is clear: if we take a periodic orbit $\hat{\Lambda}_{E}$, we have:

$$
\begin{gathered}
F_{0}\left(\hat{\Lambda}_{E}\right)=\hat{\Lambda}_{E}, \\
S_{0}\left(\hat{\Lambda}_{E}\right)=\hat{\Lambda}_{E} .
\end{gathered}
$$

These two conditions have a geometrical meaning: (8) means that $\hat{\Lambda}_{E}$ is an invariant curve of the Poincaré map, and therefore, its orbits under the continuous flow constitute a 2-torus, which is invariant under the flow. Condition (9) means that the stable and unstable manifolds of this curve only have homoclinic connections.

After the scaling done in section 2.2, we have that for high energy, or what is the same, for small $\varepsilon$, the external potential is an small and slow perturbation of the geodesic flow. Therefore, all the geometric structures that we constructed based on normal hyperbolicity and transversality persist for high energy. In particular, the manifold $\Lambda$ will persist as well as the transversality of the intersection of its stable and unstable manifolds along $\gamma$. On the other hand this perturbation is $1 / \varepsilon$-periodic in time. This will allow us to define $F, S$ analogues of the maps $F_{0}, S_{0}$, and to compute them perturbatively.

The idea now is to use the maps $F, S$ when the external forcing is acting. As we will look for orbits with growing energy, we will look for invariant curves 
of $F$, which have heteroclinic connections between them. Then we have to look for closed curves $\hat{\Lambda}_{1}, \hat{\Lambda}_{2}$ such that

$$
\begin{aligned}
F\left(\hat{\Lambda}_{i}\right) & =\hat{\Lambda}_{i}, \quad i=1,2, \\
S\left(\hat{\Lambda}_{1}\right) \cap \hat{\Lambda}_{2} & \neq \emptyset .
\end{aligned}
$$

Therefore, the main technical goal will be to compute perturbatively, for high energy, the inner and the outer maps $F$ and $S$, show that applying them alternatively we can construct heteroclinic orbits between several invariant curves, and then, show that these orbits can be shadowed by real orbits. Moreover, as we characterize the objects in $\Lambda$ by the angle $\varphi$ and the momentum $J$, which is the square root of the energy, to compute the outer map $S$, we will follow the classical Poincaré method, to measure the increment of energy during a homoclinic excursion.

\subsection{The perturbed invariant manifold. Normal hyperbolicity}

In order to study the dynamics with external potential, we consider the autonomous flow associated to the Hamiltonian (3).

$$
\begin{aligned}
\dot{p} & =-\frac{\partial H_{0}}{\partial q}(p, q)-\delta \frac{\partial U}{\partial q}(q, \varepsilon s) ; \quad \delta=\varepsilon^{2} \\
\dot{q} & =\frac{\partial H_{0}}{\partial p}(p, q) \\
\dot{s} & =1
\end{aligned}
$$

defined on the extended phase space $\mathbf{T}^{*} \mathbb{T}^{2} \times \frac{1}{\varepsilon} \mathbb{T}^{1}$.

The reason why we introduce the notation $\delta=\varepsilon^{2}$ above is so that we can study more conveniently the limit $\varepsilon \rightarrow 0$. The normally hyperbolic perturbation theory can make statements for the above system for all $\delta \leq \delta_{0}$ and one can choose $\delta_{0}$ independently of $\varepsilon$. This allows us to make statements for $\varepsilon$ small enough, even if the scaled flow is meaningless in this limit.

We note that for the unperturbed case $(\delta=0)$, we have that

$$
\tilde{\Lambda}:=\Lambda \times \frac{1}{\varepsilon} \mathbb{T}^{1} \simeq\left[J_{0}, \infty\right) \times \mathbb{T}^{1} \times \frac{1}{\varepsilon} \mathbb{T}^{1}
$$

is a 3-dimensional manifold locally invariant for this flow. This manifold is also normally hyperbolic. The next theorem assures the persistence of a hyperbolic manifold for $\varepsilon>0$ close to this one, and it relies in results of hyperbolicity theory. ${ }^{1}$ 
Theorem 4 Assume that we have a system of equations as in (10), and of class $\mathcal{C}^{r}, 2 \leq r<\infty$. Then, there exists $\varepsilon_{*}>0$ such that for $|\varepsilon|<\varepsilon_{*}$, there is $a \mathcal{C}^{r-1}$ function

$$
\mathcal{F}:\left[J_{0}+K \varepsilon^{2}, \infty\right) \times \mathbb{T}^{1} \times \frac{1}{\varepsilon} \mathbb{T}^{1} \times\left[0, \varepsilon_{*}^{2}\right) \rightarrow \mathbf{T}^{*} \mathbb{T}^{2} \times \frac{1}{\varepsilon} \mathbb{T}^{1}
$$

such that

$$
\tilde{\Lambda}_{\varepsilon}=\mathcal{F}\left(\left[J_{0}+K \varepsilon^{2}, \infty\right) \times \mathbb{T}^{1} \times \frac{1}{\varepsilon} \mathbb{T}^{1} \times\left\{\varepsilon^{2}\right\}\right)
$$

is locally invariant for the flow (10) and is $\varepsilon^{2}$-close to $\tilde{\Lambda}$ in the $\mathcal{C}^{r-2}$ sense. Moreover, $\tilde{\Lambda}_{\varepsilon}$ is a hyperbolic manifold. We can find a $\mathcal{C}^{r-1}$ function

$$
\mathcal{F}^{\mathrm{s}}:\left[J_{0}+K \varepsilon^{2}, \infty\right) \times \mathbb{T}^{1} \times \frac{1}{\varepsilon} \mathbb{T}^{1} \times[0, \infty) \times\left[0, \varepsilon_{*}^{2}\right) \rightarrow \mathbf{T}^{*} \mathbb{T}^{2} \times \frac{1}{\varepsilon} \mathbb{T}^{1}
$$

such that its (local) stable invariant manifold takes the form

$$
W^{\mathrm{s}, \text { loc }}\left(\tilde{\Lambda}_{\varepsilon}\right)=\mathcal{F}^{\mathrm{s}}\left(\left[J_{0}+K \varepsilon^{2}, \infty\right) \times \mathbb{T}^{1} \times \frac{1}{\varepsilon} \mathbb{T}^{1} \times[0, \infty) \times\left\{\varepsilon^{2}\right\}\right) .
$$

If $x=\mathcal{F}\left(J, \varphi, s, \varepsilon^{2}\right) \in \tilde{\Lambda}_{\varepsilon}$, then $W^{\text {s,loc }}(x)=\mathcal{F}^{\mathrm{s}}\left(\{J\} \times\{\varphi\} \times\{s\} \times[0, \infty) \times\left\{\varepsilon^{2}\right\}\right)$. Therefore $W^{\text {s,loc }}\left(\tilde{\Lambda}_{\varepsilon}\right)$ is $\varepsilon^{2}$-close to $W^{\text {s,loc }}(\tilde{\Lambda})$ in the $\mathcal{C}^{r-2}$ sense. Analogous results hold for the unstable manifold.

Let us observe that Theorem 4 only guarantees local invariance for $\tilde{\Lambda}_{\varepsilon}$. But, in a moment, we will show that this manifold has KAM 2-dimensional tori, that will provide with invariant boundaries for it. Therefore, it is possible to take $\tilde{\Lambda}_{\varepsilon}$ invariant. This remark leads to some extra uniqueness, etc. which could be useful for future developments. However, we will not use it in the present paper.

\subsection{The perturbed inner map. Averaging theory}

Once we have the 3 -dimensional perturbed invariant manifold $\tilde{\Lambda}_{\varepsilon}$ given by (11), we can consider the flow restricted to it, and, after some symplectic changes of variables, we can write it as a $\mathcal{C}^{r-2}$ Hamiltonian system with one and a half degrees of freedom, and $1 / \varepsilon$ periodic in the time $s$. More precisely, this Hamiltonian can be written in the local coordinates $(J, \varphi)$ of $\Lambda$ as

$$
k_{\varepsilon}(J, \varphi, \varepsilon s)=\frac{1}{2} J^{2}+\varepsilon^{2} k_{1}(J, \varphi, \varepsilon s ; \varepsilon) .
$$

The Hamiltonian $k_{\varepsilon}(J, \varphi, \varepsilon s)$ is non-autonomous, but it has an slow, periodic, dependence on the time $s$. We can apply the Adiabatic Invariance Theorem, eliminating the fast angle $\varphi$ by means of an averaging procedure up to any order $\varepsilon^{m}$. (More details on the averaging procedure can be found in ${ }^{7}$ or in ${ }^{1}$.) 
Theorem 5 Let $k_{\varepsilon}(J, \varphi, \varepsilon s)$ be the $\mathcal{C}^{r-2}$ Hamiltonian, 1-periodic in $\varphi$ and $\varepsilon s$ given in (13).

Then, for any $0<m<r-2$, there exists a canonical change of variables $(J, \varphi, s) \mapsto(I, \psi, s), 1$-periodic in $\varphi$ and $\varepsilon s$, which is $\varepsilon^{2}$-close to the identity in the $\mathcal{C}^{r-2-m}$ topology, such that transforms the Hamiltonian system of Hamiltonian $k_{\varepsilon}(J, \varphi, \varepsilon s)$ into a Hamiltonian system of Hamiltonian $K_{\varepsilon}(I, \psi, \varepsilon s)$. This new Hamiltonian is a $\mathcal{C}^{r-2-m}$ function of the form:

$$
K_{\varepsilon}(I, \psi, \varepsilon s)=K_{\varepsilon}^{0}(I, \varepsilon s)+\varepsilon^{m+1} K_{\varepsilon}^{1}(I, \psi, \varepsilon s),
$$

with $K_{\varepsilon}^{0}(I, \varepsilon s)=\frac{1}{2} I^{2}+\mathrm{O}_{\mathcal{C}^{1}}\left(\varepsilon^{2}\right)$, where the notation $\mathrm{O}_{\mathcal{C}^{1}}\left(\varepsilon^{2}\right)$ means a function whose $\mathcal{C}^{1}$ norm is $\mathrm{O}\left(\varepsilon^{2}\right)$.

Now, we consider the perturbed inner map in these new variables. First of all, let us note that the Poincaré return maps are defined in the intersection of the 3-dimensional perturbed invariant manifold $\tilde{\Lambda}_{\varepsilon}$ given by (11) with the Poincaré sections given by fixing the time $s$, that we denote $\Lambda_{\varepsilon}^{s} \subset \mathbf{T}^{*} \mathbb{T}^{2}$ :

$$
\left(\Lambda_{\varepsilon}^{s}, s\right)=\mathcal{F}\left(\left[J+K \varepsilon^{2}, \infty\right] \times \mathbb{T}^{1} \times\{s\}, \varepsilon^{2}\right) .
$$

By Theorem 4, for every $s, \Lambda_{\varepsilon}^{s}$ is $\varepsilon^{2}$-close to the unperturbed manifold $\Lambda$ in the $\mathcal{C}^{r-2}$ sense. In the variables $(I, \psi)$, we have that the perturbed inner map $F$, defined on $\Lambda$, is $\varepsilon^{m}$-close to an integrable map:

Lemma 6 In the conditions of Theorem 5, the map $F: \Lambda \rightarrow \Lambda$, which is exact symplectic, can be written in the coordinates $(I, \psi)$ as

$$
F(I, \psi)=\left(I, \psi+\frac{1}{\varepsilon} A(I, \varepsilon)\right)+\varepsilon^{m} R_{\varepsilon}(I, \psi),
$$

where $A(I, \varepsilon)=\varepsilon \int_{0}^{1 / \varepsilon} D_{1} K_{\varepsilon}^{0}(I, \varepsilon s) d s=I+\mathrm{O}\left(\varepsilon^{2}\right)$, and $R_{\varepsilon}$ is a $\mathcal{C}^{r-m-4}$ function.

Now, in these new coordinates, the KAM theorem provides the existence of invariant curves for the perturbed inner map $F$, which fill out the manifold $\Lambda$ except for gaps which are of the order of $\varepsilon^{m / 2+1}$ :

Theorem 7 Assume that $r-m-4 \geq 6$. Then, if $\varepsilon$ is small enough, for a set of $\omega$ of Diophantine numbers of exponent 5/4, we can find invariant curves $\hat{\Lambda}_{\omega}$ for $F$ which are the graph of $\mathcal{C}^{r-m-7}$ functions $u_{\omega}$, with $\left\|u_{\omega}\right\|_{\mathcal{C}^{r-m-7}} \leq$ const $\varepsilon^{m / 2}$, and the motion on them is $\mathcal{C}^{r-m-7}$ conjugate to the rotation by $\omega$. The set of these invariant curves cover the whole annulus except for a set of measure smaller than const $\varepsilon^{m / 2+1}$.

Moreover, we can find expansions $u_{\omega}=u_{\omega}^{0}+\varepsilon^{m} u_{\omega}^{1}+r_{\omega}$, with $\|r\|_{\mathcal{C}^{r-m-8}} \leq$ const $\varepsilon^{2 m}$, and $\left\|u^{1}\right\|_{\mathcal{C}^{r-m-8}} \leq$ const. 
Remark 8 Note that these KAM curves with frequency $\omega$ that we have produced for the map $F$ are really whiskered tori for the extended flow with frequencies $(\omega, \varepsilon)$. They could have been produced also by appealing to the Graff-Zehnder Theorem, but it is not direct from this theorem that the higher is the differentiability of the flow the smaller are the gaps between the tori. In any case, proceeding as in Zehnder ${ }^{8}$ we can obtain a normal form for the Hamiltonian $H_{\varepsilon}(p, q, \varepsilon s)$ in a neighborhood of these KAM tori:

$$
\mathcal{G}\left(I, a, \varphi, s, z^{\mathrm{s}}, z^{\mathrm{u}}\right)=\omega I+a+\frac{\Gamma}{2} I^{2}+\left\langle z^{\mathrm{s}}, \Omega(\varphi, s) z^{\mathrm{u}}\right\rangle+g\left(I, \varphi, s, z^{\mathrm{s}}, z^{\mathrm{u}}\right) .
$$

Such normal forms are useful later in the study of $\lambda$-lemmas in subsection 2.7.

We also call attention to a more general normal form due to Fontich and Martín ${ }^{9}$ which does not require that the motion on the tori satisfies Diophantine conditions and which is also the basis of the proof of a $\lambda$-lemma.

Remark 9 Note that KAM tori produced by Theorem 7 are of codimension 1 inside $\tilde{\Lambda}_{\varepsilon}$. If we choose a submanifold whose boundary consist on two KAM tori, this submanifold will be an invariant manifold for the extended flow. In this case the results of hyperbolicity perturbation theory can be extended to include uniqueness, as observed before.

Once we have the existence of the invariant tori of system (10) it is worthwhile to obtain some explicit approximations in the phases $(\varphi, s)$ and the value of the Hamiltonian $H_{\varepsilon}$ on it. To this end, it is convenient to write the potential as:

$$
U(q, \tau)=\bar{U}(\tau)+\tilde{U}(q, \tau)
$$

where the functions $\bar{U}(\tau)$, and $\tilde{U}(q, \tau)$ are given by

$$
\bar{U}(\tau)=\int_{0}^{1} U\left(\Lambda_{1 / 2}(\varphi), \tau\right) d \varphi, \quad \tilde{U}(q, \tau)=U(q, \tau)-\bar{U}(\tau)
$$

Lemma 10 Let $\omega$ be one of the frequencies allowed in Theorem \%. Then, in the coordinate system $\left(H_{\varepsilon}, \varphi, s\right)$, the torus of frequency $(\omega, \varepsilon)$ is the graph of a function $G(\varphi, s ; \varepsilon): H_{\varepsilon}=G(\varphi, s ; \varepsilon)$. Moreover, we can write

$$
G(\varphi, s ; \varepsilon)=\frac{\omega^{2}}{2}+\varepsilon^{2} \bar{U}(\varepsilon s)+\varepsilon^{3} \tilde{g}(\varphi, s ; \varepsilon)+\mathrm{O}_{\mathcal{C}^{r-m-8}}\left(\varepsilon^{4}\right),
$$

where $\tilde{g}(\varphi, \tau ; \varepsilon)$ is a 1-periodic in $(\varphi, \tau)$ function which verifies

$$
\omega D_{1} \tilde{g}(\varphi, \tau ; \varepsilon)+\varepsilon D_{2} \tilde{g}(\varphi, \tau ; \varepsilon)=D_{2} \tilde{U}\left(\Lambda_{1 / 2}^{q}(\varphi), \tau\right)+\mathrm{O}_{\mathcal{C}^{r-m-8}}\left(\varepsilon^{3}\right),
$$

with $\tilde{U}$ given in (16), and $\|\tilde{g}(\cdot, \cdot ; \varepsilon)\|_{\mathcal{C}^{r-m-8}}$ is bounded uniformly in $\varepsilon$. 
Furthermore, we can choose $\tilde{g}$ in such a way that $\tilde{g}=D_{2} \tilde{h}$. This $\tilde{h}$ satisfies (obviously)

$$
\omega D_{1} \tilde{h}(\varphi, \tau ; \varepsilon)+\varepsilon D_{2} \tilde{h}(\varphi, \tau ; \varepsilon)=\tilde{U}\left(\Lambda_{1 / 2}^{q}(\varphi), \tau\right)+\mathrm{O}_{\mathcal{C}^{r-m-8}}\left(\varepsilon^{3}\right),
$$

and $\|\tilde{h}(\cdot, \cdot ; \varepsilon)\|_{\mathcal{C}^{r-m-8}}$ is bounded uniformly in $\varepsilon$.

We call attention to the fact that, due to the slow dynamics, the functions $\tilde{g}(\varphi, \tau ; \varepsilon)$ and $\tilde{h}(\varphi, \tau ; \varepsilon)$ are not unique. However, as we will see later, the ambiguities only arise in subdominant terms.

\subsection{The perturbed outer map. Poincaré-Melnikov theory}

Due to the fact that $\tilde{\Lambda}_{\varepsilon}$ is a 3-dimensional hyperbolic manifold and that its 4-dimensional stable and unstable manifolds intersect in a 3-dimensional "homoclinic" manifold $\tilde{\gamma}_{\varepsilon}$ close to $\tilde{\gamma}=\gamma \times \frac{1}{\varepsilon} \mathbb{T}$, we know that any point on $\tilde{\Lambda}_{\varepsilon}$ is connected to another point through some orbit, and then it has sense to define the perturbed outer map. On the other hand, we know that $\tilde{\Lambda}_{\varepsilon}$ contains other invariant submanifolds that are the tori produced by Theorem 7 . Then, this homoclinic manifold $\tilde{\gamma}_{\varepsilon}$ will contain, among others, homoclinic trajectories to each of these torus as well as heteroclinic trajectories between some of them (those that are close enough). The fact that $\tilde{x}_{+}=S\left(\tilde{x}_{-}\right)$for two points $\tilde{x}_{ \pm} \in$ $\tilde{\Lambda}_{\varepsilon}$ is, by definition, equivalent to the existence of a point $\tilde{z} \in \mathbf{T}^{*} \mathbb{T}^{2} \times \frac{1}{\varepsilon} \mathbb{T}^{1}$, such that the trajectory $\tilde{\gamma}_{\varepsilon}(t)$ starting on it converges for $t \rightarrow \pm \infty$ exponentially fast to the trajectories $\tilde{\Lambda}_{\varepsilon}^{ \pm}(t)$ started in $\tilde{x}_{ \pm}$respectively, i.e. $\tilde{\Lambda}_{\varepsilon}^{ \pm}(0)=x_{ \pm}$, $\tilde{\gamma}_{\varepsilon}(0)=\tilde{z}$, and

$$
\operatorname{dist}\left(\tilde{\Lambda}_{\varepsilon}^{ \pm}(t), \tilde{\gamma}_{\varepsilon}(t)\right) \leq C e^{-\beta|t|} \quad \text { as } \quad t \rightarrow \pm \infty .
$$

We want to give criteria to decide whether $\tilde{x}_{+}$and $\tilde{x}_{-}$belong to different tori given by the inner map and then $\tilde{\gamma}_{\varepsilon}(t)$ is a heteroclinic connection between these tori.

As the points $\tilde{x}_{ \pm}$are in the perturbed manifold $\tilde{\Lambda}_{\varepsilon}$, they can be characterized by the angles $s, \varphi$ and the value of the perturbed Hamiltonian $H_{\varepsilon}$. Then, first we will compute $\tilde{x}_{+}=S\left(\tilde{x}_{-}\right)$in these local coordinates. From (7) we already know that $\varphi_{+}=\varphi_{-}+\Delta+\mathrm{O}\left(\varepsilon^{2}\right)$, where $\Delta=a_{+}-a_{-}$is the phase shift and (20) implies $s_{+}=s_{-}=s$, where $s$ is the time coordinate of the point $z$. Then, to relate the other coordinate of the points, we only need to compute the difference $H_{\varepsilon}\left(\tilde{x}_{+}\right)-H_{\varepsilon}\left(\tilde{x}_{-}\right)$- which is zero in the unperturbed case - and to decide whether $S\left(\tilde{x}_{-}\right)=\tilde{x}_{+}$and $\tilde{x}_{-}$lie in different tori of those produced by Theorem 7 . The computation of the difference $H_{\varepsilon}\left(\tilde{x}_{+}\right)-H_{\varepsilon}\left(\tilde{x}_{-}\right)$ can be done following the classical Poincaré-Melnikov method because we are 


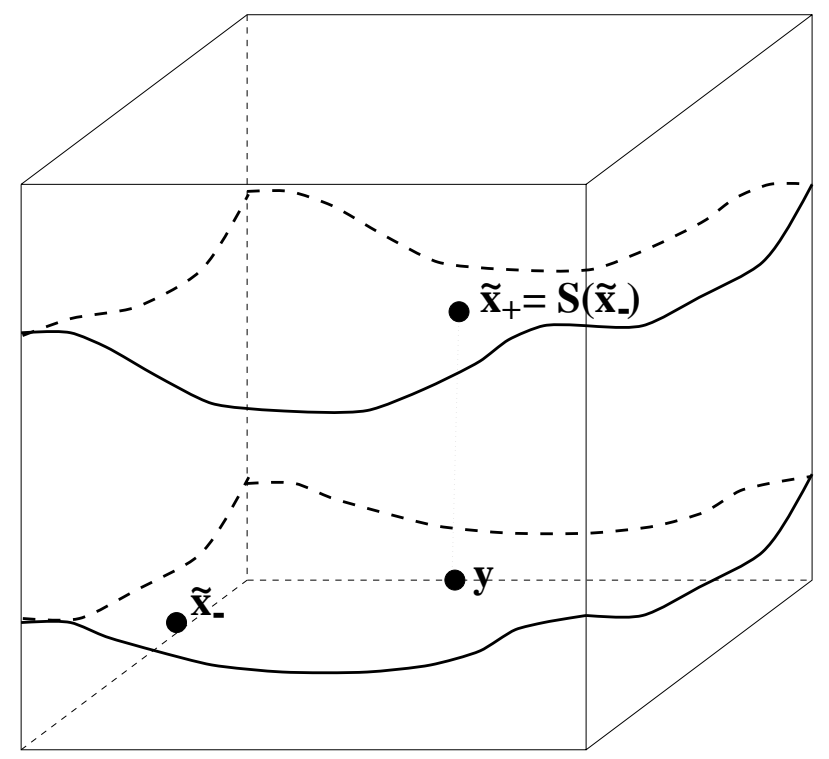

Figure 1. Illustration of the perturbed tori and the outer map: $y$ lies in the torus of $\tilde{x}_{-}$, but with the angle coordinates of $\tilde{x}_{+}=S\left(\tilde{x}_{-}\right)$.

computing the increment of the energy, which in the unperturbed system is preserved.

Since the energy $H_{\varepsilon}$ is not constant in the perturbed tori, it is not enough to compare $H_{\varepsilon}\left(\tilde{x}_{+}\right)$with $H_{\varepsilon}\left(\tilde{x}_{-}\right)$to decide whether $\tilde{x}_{+}=S\left(\tilde{x}_{-}\right)$and $\tilde{x}_{+}$lie, or not, in the same torus. Instead, we will look for the point $y$ which lies in the torus of $\tilde{x}_{-}$, but with the angle coordinates of $\tilde{x}_{+}=S\left(\tilde{x}_{-}\right)$, which are $\left(\varphi_{-}+\Delta, s\right)+\mathrm{O}\left(\varepsilon^{2}\right)$ (see Fig. 1). If $\tilde{x}_{+}$lies in the same torus as $\tilde{x}_{-}$, then $\tilde{x}_{+}=S\left(\tilde{x}_{-}\right)=y$. Then the function $H_{\varepsilon}\left(S\left(\tilde{x}_{-}\right)\right)-H_{\varepsilon}(y)$, which we will explicitly compute to first order, has to be not zero if the tori have a heteroclinic connection. In general, this function is a measure of how much the homoclinic excursion gains over just staying on the torus. It gives us a quantitative measurement of the width of the heteroclinic connections.

We can compute $H_{\varepsilon}(y)$ using the local expression of the torus that contains $\tilde{x}_{-}$, given in Lemma 10 in terms of the energy and angle coordinates. All this is summarized in the following lemma.

Lemma 11 Let $\tilde{x}_{+}$and $\tilde{x}_{-}$two points on $\tilde{\Lambda}_{\varepsilon}$, such that $\tilde{x}_{+}=S\left(\tilde{x}_{-}\right)$. Let $y$ be a point on $\tilde{\Lambda}_{\varepsilon}$, belonging to the torus that contains $\tilde{x}_{-}$, with the phases of 
$\tilde{x}_{+}$. Then

$$
\begin{aligned}
H_{\varepsilon}\left(\tilde{x}_{+}\right)-H_{\varepsilon}(y)= & \varepsilon^{3} M\left(\varphi_{0}, \varepsilon s, E ; \varepsilon\right)+\mathrm{O}\left(\varepsilon^{5}\right) \\
=\varepsilon^{3}\left(\lim _{\left(T_{1}, T_{2}\right) \rightarrow \infty} \int_{-T_{1}}^{T_{2}} d t D_{2} \tilde{U}\left(\gamma_{E}^{q}\left(t+\frac{\varphi_{0}}{\sqrt{2 E}}\right), \varepsilon s+\varepsilon t\right)\right. & \quad \tilde{g}\left(\varphi_{0}+a_{+}+\sqrt{2 E} T_{2}, \varepsilon s+\varepsilon T_{2}\right) \\
& \left.+\tilde{g}\left(\varphi_{0}+a_{-}-\sqrt{2 E} T_{1}, \varepsilon s-\varepsilon T_{1}\right)\right)+\mathrm{O}\left(\varepsilon^{5}\right),
\end{aligned}
$$

where $\tilde{x}_{+}=\left(\Lambda_{E}\left(\left(\varphi_{0}+a_{+}\right) / \sqrt{2 E}\right)+\mathrm{O}\left(\varepsilon^{2}\right), s\right), E=J_{0}^{2} / 2$, and where $\tilde{g}$ is the function given in Lemma 10 verifying (18), associated to this torus.

Then, it is clear that, if the function $M\left(\varphi_{0}, \varepsilon s, E ; \varepsilon\right)$, usually called Melnikov function, has non-degenerate zeros, the image of the torus of frequencies $(\sqrt{2 E}, \varepsilon)$ by the outer map $S$ intersects itself, or, what is the same, there exists some homoclinic orbit to it. Relatedly, this function will allow us to quantify the width of the jumps in heteroclinic connections. It so happens that the Melnikov function is the gradient of a potential - often called the Melnikov potential -10 defined by:

$$
\begin{aligned}
L\left(\varphi_{0}, \varepsilon s, E ; \varepsilon\right)=( & \lim _{\left(T_{1}, T_{2}\right) \rightarrow \infty} \int_{-T_{1}}^{T_{2}} d t \tilde{U}\left(\gamma_{E}^{q}\left(t+\frac{\varphi_{0}}{\sqrt{2 E}}\right), \varepsilon s+\varepsilon t\right) \\
& -\tilde{h}\left(\varphi_{0}+a_{+}+\sqrt{2 E} T_{2}, \varepsilon s+\varepsilon T_{2}\right) \\
& \left.+\tilde{h}\left(\varphi_{0}+a_{-}-\sqrt{2 E} T_{1}, \varepsilon s-\varepsilon T_{1}\right)\right),
\end{aligned}
$$

where $D_{2} \tilde{h}=\tilde{g}$ and $\tilde{h}$ verifies (19).

(In particular, the zeros of the Melnikov function-the homoclinic connections - correspond to the critical points of the Melnikov potential and one can bring Morse theory to bear on the problem of homoclinic connections. ${ }^{10}$ )

For the system at hand, we can take advantage of the slow dynamics and we can give an explicit formula for the leading order term of the Melnikov potential in terms of the potential $U$ and the unperturbed geodesics. This explicit expression will be given in terms of a function $\mathcal{L}(\tau)$, called Poincaré function, and will be quite important to establish that, for high enough energiesin the scaled variables for small enough $\varepsilon$ - the KAM tori have homoclinic and heteroclinic intersections.

\section{Lemma 12}

$$
L\left(\varphi_{0}, \varepsilon s, E ; \varepsilon\right)=\frac{1}{\sqrt{2 E}} \mathcal{L}\left(\varepsilon\left(s-\frac{\varphi_{0}}{\sqrt{2 E}}\right)\right)+\mathrm{O}_{\mathcal{C}^{1}}(\varepsilon),
$$




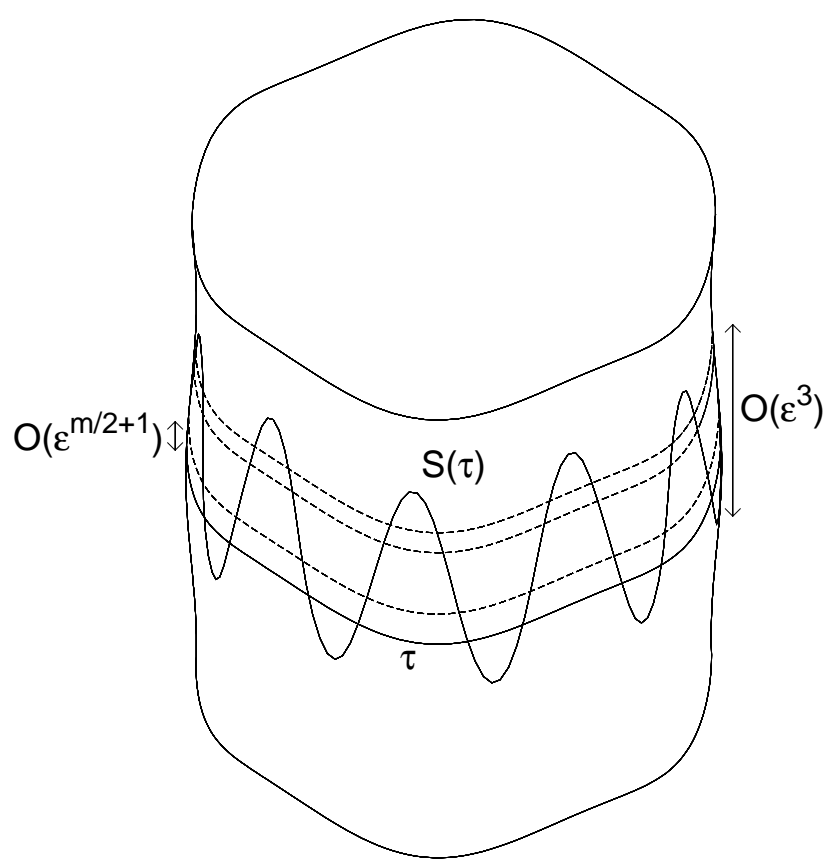

Figure 2. Ilustration of the action of the map $S$ on a torus $\tau: S(\tau)$ intersects transversally tori close to $\tau$.

where

$$
\mathcal{L}(\tau)=\lim _{\left(T_{1}, T_{2}\right) \rightarrow \infty}\left(\int_{-T_{1}}^{+T_{2}} d t \tilde{U}\left(\gamma_{1 / 2}(t), \tau\right)-\int_{-T_{1}+a_{-}}^{+T_{2}+a_{+}} d t \tilde{U}\left(\Lambda_{1 / 2}(t), \tau\right)\right) .
$$

Proposition 13 Given a metric that satisfies the genericity conditions of Theorem 2, and orbits $\Lambda_{1 / 2}, \gamma_{1 / 2}$ of the geodesic flow as in the conclusion of Theorem 2, the set of periodic potentials for which the Poincaré function $\mathcal{L}(\tau)$ is identically constant is a $\mathcal{C}^{l}$ closed subspace of infinite codimension for $l>0$.

\subsection{Transition chains and shadowing orbits}

In this section we will see that, if the Poincaré function $\mathcal{L}(\tau)$ is non-constant, the perturbed invariant tori have heteroclinic intersections if $1+m / 2>3$, that is, if the measure of the gaps between them is smaller than the jumps produced by the homoclinic excursions (see Figure 2). This give us the existence of a so called transition chain. After this, some results on the literature and some 
point set topology, will give us the existence of orbits that follow an infinite chain of these tori.

Lemma 14 Assume that $r \geq 15$. If the function $\mathcal{L}(\tau)$ is not constant, we can find $K>0$ such that for $\varepsilon$ sufficiently small, given a $K A M$ torus $\mathcal{T}$, we can find other $K A M$ tori $\mathcal{T}^{+}, \mathcal{T}^{-}$such that

$$
\begin{aligned}
W_{\mathcal{T}}^{\mathrm{u}} \pitchfork W_{\mathcal{T}^{+}}^{\mathrm{s}}, \quad W_{\mathcal{T}}^{\mathrm{u}} \pitchfork W_{\mathcal{T}^{-}}^{\mathrm{s}}, \\
\min H_{\varepsilon}(\mathcal{T}) \geq \max H_{\varepsilon}\left(\mathcal{T}^{-}\right)+K \varepsilon^{3}, \\
\max H_{\varepsilon}(\mathcal{T}) \leq \min H_{\varepsilon}\left(\mathcal{T}^{+}\right)-K \varepsilon^{3} .
\end{aligned}
$$

Remark 15 The lemma above does not assert the existence of transverse homoclinic orbits to any of the tori $\mathcal{T}, \mathcal{T}^{-}$and $\mathcal{T}^{+}$. The existence of transverse homoclinic orbits is related to the existence of nondegenerate critical points of the Poincaré function. We emphasize that, for our purposes, what we need are transverse heteroclinic intersections.

Our next goal is to see that the heteroclinic orbits that can be obtained by Lemma 14 can be shadowed by true orbits of the system. As it is usual in the literature for Arnold diffusion, the key step is to find an appropriate inclination lemma (also called sometimes $\lambda$-lemma).

In the literature, one can find very sharp inclination lemmas - including even some estimates of the times needed to do the shadowing - for analytic maps, when the rotation is Diophantine. ${ }^{11,12,13}$. The result that we have found best adapted to our purposes is that of Fontich and Martín ${ }^{9}$ for whiskered tori with one dimensional strong (un)stable directions - as is the case in the problem we are considering-, which works for $\mathcal{C}^{1}$ maps and only requires that the torus has an irrational rotation.

A particular case of these results ${ }^{9}$ is:

Lemma 16 Let $f$ be a $\mathcal{C}^{1}$ symplectic mapping in a $2(d+1)$ symplectic manifold. Assume that the map leaves invariant a $\mathcal{C}^{1} d$-dimensional torus $\mathcal{T}$ and that the motion on the torus is an irrational rotation. Let $\Gamma$ be a $d+1$ manifold intersecting $W_{\mathcal{T}}^{\mathrm{u}}$ transversally.

Then

$$
W_{\mathcal{T}}^{\mathrm{s}} \subset \overline{\bigcup_{i>0} f^{-i}(\Gamma)} .
$$

An immediate consequence of this is that any finite transition chain can be shadowed by a true orbit. The argument for infinite chains requires some elementary point set topology.

Lemma 17 Let $\left\{\mathcal{T}_{i}\right\}_{i=1}^{\infty}$ be a sequence of transition tori. Given $\left\{\varepsilon_{i}\right\}_{i=1}^{\infty} a$ sequence of strictly positive numbers, we can find a point $P$ and a increasing 
sequence of numbers $T_{i}$ such that

$$
\Phi_{T_{i}}(P) \in N_{\varepsilon_{i}}\left(\mathcal{T}_{i}\right),
$$

where $N_{\varepsilon_{i}}\left(\mathcal{T}_{i}\right)$ is a neighborhood of size $\varepsilon_{i}$ of the torus $\mathcal{T}_{i}$.

From Lemma 14 and Lemma 17, we obtain the following result, which clearly implies Theorem 1 .

Theorem 18 Assume that the metric $g$ satisfies the assumptions of Theorem 2 and that $U$ is such that the Poincae function $\mathcal{L}$ is not constant. Assume moreover that both $g$ and $U$ are $\mathcal{C}^{15}$. Then, there exist $M>0, \alpha>0$, such that if

$$
I_{i}=\left[E_{-}^{i}, E_{+}^{i}\right] \quad i=1, \ldots
$$

is any sequence of intervals such that

$$
\begin{aligned}
E_{-}^{i} & \geq M \\
\left(E_{+}^{i}-E_{-}^{i}\right) & \geq M\left(E_{+}^{i}\right)^{-\alpha} .
\end{aligned}
$$

Then, we can find an orbit $p(t), q(t)$ of the Hamiltonian flow and an increasing sequence of times $t_{1}<t_{2}<\cdots<t_{n}<\cdots$, such that

$$
H\left(p\left(t_{i}\right), q\left(t_{i}\right), t_{i}\right) \in I_{i} .
$$

and $\left(p\left(t_{i}\right), q\left(t_{i}\right)\right)$ is in a neighborhood of size $M\left(E_{-}^{i}\right)^{-2}$ of the periodic orbit $\Lambda_{E_{-}^{i}}$.

Remark 19 By assuming more differentiability in the hypothesis of the theorem, we can get $\alpha$ to be arbitrarily large. This is because, if the system is sufficiently differentiable, we can perform more steps of averaging theory so that the KAM tori are much closer.

Remark 20 Observe that the existence of the symbolic dynamics above establishes that the set of orbits that have unbounded energy is uncountable. We have uncountably many choices of energy ranges to hit. By going over the argument with somewhat more care we can see that there are different orbits that realize these choices.

\section{Some final remarks}

We conclude with some final questions and remarks about two problems that have been asked us and that are relevant from the physical interpretation of the problem.

The most important question is whether the mechanism presented here is the only one or even the dominant one. All the questions that we present 
here have a version for the orbits constructed here and for the set of orbits whose energy grows to infinity.

Q1: What is the measure of the set of orbits whose energy goes to infinity (resp. those constructed here)?

We know nothing about this question except to point out that using the symbolic dynamics, the set of orbits constructed here is uncountable. Presumably, the set of orbits constructed here is of zero measure.

Q2: What is the fastest rate of growth of the energy one can achieve?

The following upper bound is elementary.

Proposition 21 If $q(t)$ is a solution of the Hamiltonian flow of $H(p, q, t)=$ $\frac{1}{2} g_{q}(p, p)+U(q, t)$, then

$$
|H(p(t), q(t))| \leq K t
$$

Proof. Note that

$$
\frac{d}{d t} H(p(t), q(t))=\frac{\partial}{\partial t} U(q(t), t),
$$

and the right hand side is uniformly bounded since it ranges over $\mathbb{T}^{2} \times \mathbb{T}$.

One can estimate heuristically the rate of growth of the energy of the solutions we constructed as follows: Note that the mechanism that makes the energy grow is the energy gained in the heteroclinic excursion. As we have seen, the scaled energy gained in one heteroclinic excursion is $\approx E^{-3 / 2}$, this corresponds to a gain of physical energy of $\approx E^{-1 / 2}$ but in a unit of time, we can make $\approx E^{1 / 2}$ excursions.

Hence, in a unit of time the energy gained by the mechanism proposed before is constant, hence, we expect that the orbits have energy that grows about linearly: $E(t) \approx t$.

The above argument can be turned into a rigorous proof of the speed of diffusion of pseudo-orbits with arbitrarily small (but non-zero) error.

The reason why the above argument cannot be turned into a rigorous proof for orbits is because there is no reason why an orbit that shadows $N$ jumps needs to spend only a fixed amount of time near each torus. Indeed, the proofs available in the literature require that $N$ jumps spend a significantly longer time.

The reason for that is that in order to do $N$ jumps, the first jumps need to be planned with a greater accuracy and one requires to spend much longer near the torus in order to wait for the very delicate window of opportunity for the very accurate jump. 
It seems to be unknown whether this is really necessary or whether it is just an artifact of the method of proof. We point out that, in a very similar problem, Chirikov conjectured in a related case that indeed one would not need to increase the time spent near the tori for a long shadowing chain. Hence, if the Chirikov conjecture were true for our case (it seems to us that in our case the conjecture is more plausible than in the case originally stated for), then we could get a rate of escape that agrees with the upper bound up to a finite constant.

Q3: Is there a quantum mechanical analogue of the phenomena discovered here?

Of course, we consider as the quantum mechanical model the Hamiltonian given by the Laplacian and a time periodic potential.

We note that an analogue of Proposition 21 can be proved in quantum mechanics, from

$$
\frac{d}{d t}\langle\Psi|H| \Psi\rangle=\left\langle\Psi\left|\frac{\partial V}{\partial t}\right| \Psi\right\rangle .
$$

One is tempted to argue that the construction presented in the previous sections is too delicate to survive the quantum mechanical effects of uncertainty etc.

We would like to decide whether there are analogues of the existence of orbits whose energy goes to infinity and also an analogue of the symbolic dynamics result.

Note that in quantum mechanics, as soon as we construct one solution whose energy grows to infinity, we have an open and dense set of solutions whose energy grows to infinity.

\section{Acknowledgements}

We thank J.N. Mather for communicating his results and for encouragement. This work has been partially supported by the NATO grant CRG950273. Research by A.D. and T.M.S. is also supported by the Spanish grant DGICYT PB94-0215, the Catalan grant CIRIT 1996SGR-00105, and the INTAS project 97-10771. Research by R.L. is also supported by NSF grants. We also thank TICAM, UPC and IMA for invitations that made possible these collaborations. 


\section{References}

1. A. Delshams, R. de la Llave, and T.M. Seara. A geometric approach to the existence of orbits with unbounded energy in generic periodic perturbations by a potential of generic geodesic flows of $\mathbb{T}^{2}$. Preprint 98-591, mp_arc@math . utexas . edu, 1998.

2. J.N. Mather. Graduate course at Princeton, 95-96, and Lectures at Penn State, Spring 96, Paris, Summer 96, Austin, Fall 96.

3. L. Chierchia and G. Gallavotti. Drift and diffusion in phase space. Ann. Inst. H. Poincaré Phys. Théor., 60(1):1-144, 1994.

4. S. Bolotin and D. Treschev. Unbounded growth of energy in nonautonomous Hamiltonian systems. Nonlinearity, 12(2):365-388, 1999.

5. V.I. Arnold. Instability of dynamical systems with several degrees of freedom. Sov. Math. Doklady, 5:581-585, 1964.

6. V.I. Arnold and A. Avez. Ergodic problems of classical mechanics. Benjamin, New York, 1967.

7. V.I. Arnold, V.V. Kozlov, and A.I. Neishtadt. Dynamical Systems III, volume 3 of Encyclopaedia Math. Sci. Springer, Berlin, 1988.

8. E. Zehnder. Generalized implicit function theorems with applications to some small divisor problems, I and II. Comm. Pure Appl. Math., 28(1):91-140, 1975, and 29(1):49-111, 1976.

9. E. Fontich and P. Martín. Arnold diffusion in perturbations of analytic integrable hamiltonian systems. Preprint 98-319, mp_arc $@$ math.utexas. edu, 1998.

10. A. Delshams and R. Ramírez-Ros. Melnikov potential for exact symplectic maps. Comm. Math. Phys., 190:213-245, 1997.

11. J.P. Marco. Transition le long des chaînes de tores invariants pour les systèmes hamiltoniens analytiques. Ann. Inst. H. Poincaré Phys. Théor., 64(2):205-252, 1996.

12. Jacky Cresson. A $\lambda$-lemma for partially hyperbolic tori and the obstruction property. Lett. Math. Phys., 42(4):363-377, 1997.

13. Enrico Valdinoci. Whiskered transistion tori for a priori stable and unstable hamiltonian systems. Preprint 98-200, mp_arc@math.utexas . edu, 1998. 